\title{
Finzioni e funzioni del sogno: la costante onirico-ideologica nella lirica occitana tra cinque e novecento
}

\author{
Roberta Capelli
}

Università di Verona

\begin{abstract}
L'articolo prende in esame gli autori occitani moderni e contemporanei che hanno utilizzato sistematicamente il tema poetico del sogno attribuendogli la funzione ideologica di strumento per la rivendicazione politico-letteraria di riscoperta ed esaltazione del passato, specificamente dell'eredità trobadorica, in quanto epoca d'oro dell'indipendenza linguistica, economica e artistica del Midi della Francia. Da Jean de Nostredame, precursore erudito della rinascenza provenzale del XVI secolo, fino al contemporaneo Max Rouquette, passando per l'opera mediatrice primo ottocentesca di Fabre d'Olivet e il patriottismo sentimentale di Frédéric Mistral (Premio Nobel per la Letteratura nel 1904), l'analisi sincronica e diacronica del presente contributo evidenzia le caratteristiche fondamentali di ciascun autore in rapporto alla tendenza generale di una poesia «impegnata», dove l'elemento onirico è usato come parentesi meta-narrativa all'interno del discorso poetico, concepito per accogliere le riflessioni del poeta sulla sua missione nella e per la Storia, in forma di immagine atemporale, collettiva, universalizzante.
\end{abstract}

Parole chiave: trovatori, Medievalismo, Renaissance Provençale, Felibrismo, Corti d'Amore, sestina.

\begin{abstract}
This essay studies the Provençal authors who systematically exploited the dream motif for ideological purposes, taking on the legacy of the Troubadours in order to reassert the strong sense of linguistic and literary commonality between regions in Southern France, which creates difficult issues of competition between the literature in the Provençal mother tongue and the homogenizing power of the French language and the government's centralizing influence. The analysis focuses upon the erudite approach of the $16^{\text {th }}$ century forerunner Jean de Nostredame, the pre-Romantic taste of Antoine Fabre d'Olivet (1767-1825), the Romantic demands of Frédéric Mistral (Nobel Prize for Literature, 1904), and the recent experiments by Max Rouquette (who died 2005). By examining these literary interpretations of the dream theme, it is therefore possible to highlight the common narrative and lyrical functions they fulfil synchronically and diachronically: the reverie appears to be a metadiegetic digression which brings poetic discourse to a halt and is conceived to express the poet's universalizing meditations on his historical, cultural and artistic mission.
\end{abstract}

Key words: Troubadours, Medievalism, Renaissance Provençale, Felibrism, Courts of Love, sextine. 
Nella periodizzazione a maglia larga proposta dal titolo di questa indagine, la selezione e particolarizzazione del motivo onirico delinea due fondamentali filoni tra loro tipologicamente molto diversi, l'uno all'insegna della soggettività estetizzante del sogno in quanto sfogo dell'anima, l'altro invece teso alla sua oggettivazione ideologicamente connotata. E se, nel primo caso, la campionatura dovrà tener conto di una varietà di forme e stili mutevoli per cifra stilistica d'autore all'interno di mode d'epoca (all'insegna, dunque, di un'esaltazione della «singolarità» ed "eccezionalità» della componente individuale, centrifuga rispetto alle schematizzazioni critiche d'assieme), nel secondo caso — quello su cui intendo concentrarmi- è possibile individuare una sorta d'ideale linea di sviluppo diacronico a base tematico-ideologica comune a generazioni successive di intellettuali/letterati.

Il percorso progressivo di codificazione formale e funzionale del sogno di matrice - per così dire- engagée s'inaugura con Jean de Nostredame in piena temperie petrarchista, attraversa il genre troubadour settecentesco con Fabre d'Olivet e il Romanticismo con Mistral, per approdare agli esiti ultimi dello sperimentalismo concettuale di Max Rouquette. Si tratta ovviamente di una galleria per specimina di massima rappresentatività, anche se, in effetti, poiché la letteratura di espressione occitana riguadagna parte della dignità identitaria persa con l'editto di Villers-Cottêrets (1539) solo con l'ondata di Medievalismo ottocentesco, gli isolati Nostredame e Fabre d'Olivet si sono da tempo meritati l'eloquente titolo di "precursori dei Felibri». ${ }^{1}$

Come necessaria - e in questa sede sintetica ${ }^{2}$ - premessa storico-letteraria andrà ricordato l'inarrestabile declino della lingua d'oc dalla Crociata contro gli Albigesi (1208-1229) e conseguente diaspora trobadorica in Italia, fino al summenzionato editto di Villers-Cottêrets che impone l'uso esclusivo del francese in campo amministrativo e giuridico. Ridotte le varietà occitane al rango di dialetti e schiacciata la produzione in patois dall'influenza centralizzatrice della capitale, il Midi entra nella periferia culturale delle aree marginali e marginalizzate, costantemente in bilico tra orgogliosa chiusura nazionalistica e rivendicazioni socio-linguistiche a base artistica, tra valorizzazione dei generi popolari $\mathrm{o}$ adesione attardata a modelli europei già ampiamente sfruttati, e impegno alla difficile rivitalizzazione creativa di un'identità meridionale.

Nello specifico del nostro tema, si osserva quindi, da un lato, la ripresa nemmeno tanto mascherata delle chimere oniriche di maniera tipiche del Barocco (penso, soprattutto, alla generazione di poeti guasconi seicenteschi - Bertrand Larade, Pierre Godolin e André Du Pré- informata dalla Pléiade, da Ronsard e da Desportes), ${ }^{3}$ e il decorativismo onirofantastico del neo-goti-

1. La definizione è ripresa dall'ancor oggi imprescindibile libro di Frédéric DONNADIEU, Les précurseurs des Félibres (1800-1855), Parigi, 1888 [rist. Raphèle-lès-Arles, Marcel Petit, 1980].

2. Per un inquadramento del problema rimando a Robert LAFOnT e Christian ANATOLE (a cura di), Nouvelle histoire de la littérature occitane, vol. 1, Paris: Presses Universitaires de France, 1970 , p. 275-76.

3. Sul Petrarchismo occitano, rimando al mio contributo "Anas vous en pauras rymas dolentas»: frammenti di Petrarchismo nei falsi trobadorici di Jean de Nostredame, in Amedeo QuON- 
co (e mi riferisco, in particolare, a certo Felibrismo sensibile alla lezione dell'Hugo di Odes et Ballades [1828] o agli Emaux et Camées di Théophile Gautier [1852]); $;^{4}$ dall'altro, invece, si rileva un uso diffuso del sogno come metafora o allegoria di un presente moderno in cerca di legittimazione politica e culturale, sul modello del fulgido - e volutamente idealizzato- mondo antico delle corti medievali. ${ }^{5}$ In questi autori, la psicologia del sogno non va disgiunta dall'esegesi storico-critica, e l'elemento lirico-onirico apparentemente personale e sentimentale ha sempre una componente di fondo (più o meno consapevole e sviluppata) collettiva e storicizzante, attenta a scegliere quei "frammenti storici che, a seguito della loro densità affettiva, ma soprattutto simbolica, si imprimono particolarmente nella fantasia collettiva, e li condensa in immagini atemporali, in rappresentazioni dell'essenza di quel dato gruppo umano o in archetipi dell'esistenza umana». ${ }^{6}$

Lungo l'asse Nostredame (1522-1577) - Rouquette (1908-2005) il sogno svolge pertanto una funzione di propaganda ideologica della Renaissance provençale sotto mentite spoglie letterarie, o meglio, sotto "copertura» retoricolirica di digressione di tipo ecfrastico: la natura parentetica, meta-narrativa dell'inserto onirico - sia in contesto prosastico che poetico- permette la condensazione simbolica del messaggio autoriale (soggettivo) e del dato storico (oggettivo) in una formula autoritativa ultrasintetica, in tutto simile alla rivelazione di una verità — assoluta, unilaterale, patriottica — da dispensare al pubblico dei lettori, sostenitori della causa occitana.

Il punto di partenza e irradiazione di questa verità rivelata è Jean de Nostredame, ${ }^{7}$ autore delle Vies des plus célèbres et anciens poètes provençaux (Lione

DAM (a cura di), Il Petrarchismo. Un modello di poesia per l'Europa, Atti del Convegno internazionale di studi (Bologna, 6-9 ottobre 2004), vol. II, Roma: Bulzoni, 2007, p. 441-55; in particolare la bibliografia citata alla nota 1 . Per un inquadramento tematico della produzione barocca francese, cfr. Gisèle MATHIEU-CASTELLANI, Mythes de l'éros baroque, Paris: PUF, 1981.

4. Non particolarmente feconda né esauriente la bibliografia su questo argomento; rimando a Janine R. DAKYNS, The Middle Ages in French Literature (1851-1900), London: Oxford University Press, 1973; Dorothy DOOLITTLE, The Relations between Literature and Mediaeval Studies in France from 1820 to 1860, Pennsylvania: Dissertation at the Bryn Mawr College, 1933; Emile RIPERT, La renaissance provençale (1800-1860), Paris: Hachette, 1968; Paul ZumTHOR, Le Moyen Age de Victor Hugo, in Littérales, n. 6, 1990, p. 117-24.

5. Cfr. Jacques DE CALUWÉ, Le Moyen Age littéraire occitan dans l'oeuvre de Frédéric Mistral. Utilisation éthique et esthétique; René LANSON, Le goût du Moyen Age en France au XIIIe siècle, Paris-Bruxelles: Vanoest, 1926.

6. Cfr. Eugen Drewermann, Psicologia del profondo e esegesi. 1. La verità delle forme. Sogno, mito, fiaba, saga e leggenda, Brescia: Queriniana, 1996, p. 91.

7. Per una cronologia aggiornata delle vicende biografiche di Jean de Nostredame, cfr. Jean-Yves CASANOVA, Historiographie et littérature au XVIe siècle en Provence: l'euvre de Jean de Nostreda$m e$, Thèse pour le Doctorat dès Lettres, dir. Philippe Gardy, Université Paul Valéry-Montpellier III, a.a. 1990. Dello stesso autore si vedano anche i due contributi Les sonnets de Jean de Nostredame: palimpseste et formes inavouées, in Revue des Langues Romanes, 94, n. 2, 1990 , p. 219-36, e Doas notícias istoricas de Joan de Nostradama dins sei "Mémoires historiques»: "La Guerra d'Arles» e un sirventés de Bertrand de Lamanon, in Tèxtes Occitans, 1, 1996, p. 7-28. 
1575), un'antologia «filologicamente scorretta» che raccoglie biografie (in parte inventate) e componimenti trobadorici (non di rado apocrifi), piegati a fini encomiastici (illustri genealogie vengono, ad esempio, create su misura per famiglie in vista e papabili protettori dell'ambiente lionese). Si tratta di un falso letterario che, sfruttando la novità della materia occitana all'epoca caduta quasi del tutto nell'oblìo, è destinata a diventare opera di riferimento indiscussa fino all'inizio del XIX secolo, e ad esercitare un'influenza spropositata nella propagazione di quelli che verranno canonizzati come topoi dalla produzione successiva in lingua d'oc; su tutti, il mito delle Corti d'Amore, tribunali preposti alla risoluzione delle dispute di carattere amoroso, presieduti da nobili dame di Provenza:

Les tensons estoyent disputes d'Amours qui se fasoyent entre les chevaliers et dames poëtes, entreparlans ensemble de quelque belle et subtille question d'amours, et où ils ne s'en pouvoyent accorder, ils les envoyoyent pour en avoir la diffinition aux dames illustres presidentes, qui tenoyent Cour d'Amour ouverte et planiere à Signe et à Pierrefeu, ou à Romanin, ou à autres, et là dessus en faisoyent arrests, qu'on nommoit lous Arrests d'Amours. [Vies, p. 11]

L'invenzione nostradamica assurge al rango di auctoritas, destinata ad avere grande fortuna come citazione comprobante il prestigio della lingua e letteratura del Midi, e si adatta ottimamente ai parametri psico-retorici della rilettura e riscrittura onirica: innesca — con fusione della brevitas dell'inciso e della pregnanza dell'immagine archetipica- un meccanismo proprio del sogno, cioè quello di regressione (all'Età dell'Oro medievale) / progressione (alla rinascente cultura d'oc), culminante nell'unificazione temporale di presente-passatofuturo nell'esemplarità sempre valida del mito (delle Corti d'Amore) e nella sua apertura teleologica all'interscambio tra scopo individuale (autoriale) e scopo collettivo (del pubblico).

Questo tipo di rievocazione poetica mitizzata del passato provenzale con valore esemplare in alternativa oppositiva (implicito-metaforica) alla dominante cultura/letteratura francese, trova una formulazione letterale nel pamphlet dal titolo: Réflexions sur le libelle intitulé "Lettre critique De Sextius le Salien à Euxenus le Marsellois»..., ${ }^{9}$ pubblicato da Pierre Galaup de Chasteuil nel 1702, a sostegno dei meriti socio-letterari dei trovatori e contro il loro detrattore, Pierre Joseph de Haitze. ${ }^{10}$ Il Galaup sceglie la via dell'inattaccabilità della rive-

8. L'edizione di riferimento è quella moderna, curata da Camille Chabaneau e pubblicata con introduzione e commento da Joseph Anglade, Paris, 1913 [Genève, Slatkine Reprints, 1970].

9. REFLEXIONS | SUR LE LIBELLE INTITULE | LETTRE CRITIQUE | De Sextius le Salien à Euxenus le Marsellois, Touchant le Discours sur les Arcs Triomphaux, dressez en la Ville d'Aix, à l'heureuse arrivée | de Monseigneur le Duc de Bourgo-|gne, \& de Monseigneur de Berry. | A.M.D.S.C.D.R.A.P. D.P. | A COLOGNE | Chez PIERRE LE BLANC, à | l'Enseigne de la Coronne | M.D.CCII.

10. Su questa polemica, rinvio al mio contributo Trovatori «loüables» $O$ «volages»? La controversia di inizio Settecento tra Pierre Galaup de Chasteuil e Pierre-Joseph de Haitze, in Il discorso polemico. Controversia, invettiva, pamphlet, Atti del XXXIII Convegno Interuniversitario (Brixen, 7-10 luglio 2005), Padova: Esedra, p. 205-216. 
lazione ispirata onirico-fantasmatica per la dimostrazione apodittica e autoreferenziale delle proprie tesi, difendendo cioè la validità della propria fonte (Jean de Nostredame) attraverso le ragioni addotte da quella fonte medesima (le Vies), solo camuffata da testimonianza di un "terzo-esterno» (fittizio) che è, appunto, il trovatore-messaggero del sogno, Guillaume Durand:

Dans le tems que je fasois telles ou semblables reflexions, je me couchai, \& m'êtant endormi, je me trouvai transporté en songe aux pieds du Mont Parnasse [...]. Et ce fut alors qu'un homme de fort bon air s'avançant vers moi me dit en m'abordant, je vois ce qui te travaille mon ami, \& je [...] t'éclaircirai volontiers de tout ce que tu souaites: je jettay les yeux sur cet homme, $\&$ il ne me fut pas difficile de connoître au portrait qu'en ont fait les deux Nostradamus, que c'estoit Guillaume Durand. [...] Mais je Vous prie Monsieur de m'apprendre si les Nostradamus ont dit vrai, lors qu'ils ont écrit que la Comtesse de Die s'êtoit retirée avec sa mere [...] à l'Abbaye de Saint Honoré de Tarascon? si cette Dame étoit Presidente de la Cour d'Amour? si ce Parlement étoit effectif?... [Réflexions, p. 28-34]

Il passo da sognare una Provenza mitica a topicizzare il mito di una Provenza da sogno ${ }^{11}$ è minimo e, difatti, avviene alla fine del secolo, grazie alla bizzarra figura di erudito, linguista e storico delle religioni, che è Antoine Fabre d'Olivet, lettore e ammiratore delle Vies di Jean de Nostredame, autore egli stesso di un falso letterario intitolato Le Troubadour. Poésies occitaniques du XIII siècle (Parigi 1803-1804), ${ }^{12}$ nel quale tenta di spacciare per originali e, dunque, medievali le proprie creazioni liriche di inequivocabile goût troubadour. Quello che però ci interessa è che egli opera — e apertamente dichiara- la sovrapposizione della dimensione onirica ideale con quella del locus amoenus di classica memoria, riproponendo il Midi passato e perfetto sotto forma di «rêverie historique»:

...je parcourais en observateur la patrie de nos vieux Troubadours, assis sur les mêmes rochers où il s'étaient assis, debout sur les mêmes rives, en face de la même nature, vigoureuse et sauvage, outant, pour ainsi dire, l'écho de leurs chansons romantiques, et recueillant de la bouche même de laurs descendants les reste précieux de leur langue oubliée. [...] Voilà comment, entraîné par une rêverie historique, la tête baissée et l'œil fixé sur les rochers verdoyants d'où sortent avec fracas les sources de l'Hérault, je me trouvai, du pays des Troubadours, transporté dans celui des Bardes. ${ }^{13}$

11. Cfr. Robert Lafont, Mistral et le mythe des Cours d'Amour, in Moyen Age et Littérature Comparée, Actes du VIIe Congrès national (Poitiers, 27-29 mai 1965), Paris: Didier, 1967, p. 183-96.

12. Antoine Fabre d'Olivet, Le Troubadour, poésies occitaniques du XIIIe siècle, traduites et publiées par Fabre d'Olivet, 2 vol., Paris: Henrics, 1803-1804. Poi ripubblicato come terzo volume di ID., La langue d'oc rétablie dans ses principes constitutifs, 3 vol., a cura dell'Association Fabre d'Olivet, Ganges: Steinfeld, 1992, da cui si cita.

13. Antoine Fabre d'OlIVet, La langue d'oc rétablie, op. cit., Avant-propos, vol. I, p. XXXVIII-XXXIX. 
Questa dichiarazione programmatica è tradotta in versi nel Lay d'Amistad, un'egloga-bergerie che, già come genere in sé, si dimostra estranea alla tradizione trobadorica, ma rispondente all'orecchio del tempo e allo spirito celebrativo dell'autore:

E s'el plouro un de sous moutoùs, en liogo d'un li'n torno dous.

Souven, quand çai vén un Troubaire, un Jounglar, mage en Gai-sabèr qu'ensajo de tout soun poudèr a plaire!

\section{[Le Troubadour, vv. 23-28]}

Il falso di Fabre d'Olivet viene smascherato dall'occhio acuto del filologo François Raynouard, ${ }^{14}$ uno dei maggiori rappresentanti dell'allora nascente (con le lezioni sulla poesia europea, in particolare quella neolatina, tenute da August-Wilhelm Schlegel a Berlino nel 1804, e la fondamentale Grammatik der romanischen Sprachen di Friederich Diez, Bonn 1836-1843) Provenzalistica d'impostazione scientifica; ${ }^{15}$ ma sarebbe sbagliato pensare che il diffondersi della ricerca filologica in àmbito romanzo e la sua ufficializzazione come disciplina universitaria abbia penalizzato i voli pindarici della fantasia e poesia contemporanee. $\mathrm{Al}$ contrario, lo studio sempre più approfondito delle fonti dirette e le sempre più numerose edizioni di trovatori hanno avuto l'effetto di produrre una generale rivalutazione del passato nazionale, con spiccata propensione per un revival medievale sentito come «désir de ressourcement dans l'origines. ${ }^{16}$

Non è un caso che Frédéric Mistral, il padre-fondatore del Felibrismo, il cantore appassionato del Midi rurale e puro, visto come il mondo degli affetti familiari, dello spirito patriottico, e come il luogo del ricordo nostalgico di un'indipendenza e grandezza letterarie perdute, non solo non voglia ammettere l'anacronistica paternità nostradamica delle Corti d'Amore (nonostante le ripetute sollecitazioni dell'amico-filologo Paul Meyer), ${ }^{17} \mathrm{ma}$ faccia dei Parlamenti di Dame una delle trasposizioni onirico-metaforiche più frequenti e meglio sviluppate all'interno di tutta la propria produzione letteraria. La scelta di collocare un componimento dall'eloquente titolo di

14. Raynouard, nel suo articolo del 1824 pubblicato sul Journal des Savants svela la falsificazione di Fabre d'Olivet, mostrando come l'autore abbia manipolato componimenti trobadorici originali contenuti nell'attuale ms. fr. 856 della Bibliothèque Nationale di Parigi.

15. Per una panoramica dello sviluppo della Filologia romanza può essere utile la sintesi tracciata nel cap. II: Sotto il segno della storia, p. 43-70, di Lorenzo RENZI (a cura di), Nuova introduzione alla filologia romanza, Bologna: Il Mulino, 1994.

16. Gérard Gengembre, Le Romantisme, Paris: Ellipses, 1995, p. 15.

17. Lo si ricava dagli scambi epistolari tra i due amici, raccolti da Jean Boutière, Correspondance de Mistral avec Paul Meyer e Gaston Paris, Paris: Didier, 1978. 
Roumanin ${ }^{18}$ e dall'ancora più eloquente dedica in esergo proprio a Paul Meyer nella sezione dei Pantai [= dei «Sogni»] della raccolta Lis Isclo d'Or ${ }^{19} \mathrm{mi}$ sembra confermare la traiettoria esegetica ideologico-diacronica fin qui seguita:

\begin{abstract}
...escalère, escalère, e fuguère au castèu, ilustre e desoula, de Roumanin. O glòri, o festo d'autre-tèms! O fougau de belòri, Court d'amour assetado à l'uba di calanc, tout es mut, tout es mort...
\end{abstract}

[vv. 8-12]

Attingendo al repertorio d'immagini archeologico-sentimentale e ad un lessico fortemente enfatizzato in senso crepuscolar-notturno tipici di quel Romanticismo europeo amante delle atmosfere rarefatte con rovine (Uhland e Heine in Germania, Carducci e Boito in Italia, Chateaubriand in Francia), Mistral ci introduce in un mondo che appare fatiscente e desolato (v. 22: «li rouino», v. 12: «mut» e «mort») sotto la luce del tramonto (v. 31: «lou vèspre», v. 84: «tremount dóu soulèu», v. 132: «lou calabrun»), dove però le ombre si fanno spiriti dell'oltre-tomba per chi li sa vedere con gli occhi dell'immaginazione:

Sus lou vèspre venian; li serre tranquilas escampavon en plen soun oumbro au castelas. "Faneto! iéu cridère, o gènto e bono damo, dou mounde segrenous ounte sias, se i'a d'amo...»

$$
\text { [vv. 31-34] }
$$

L'equazione meta-letteraria Mistral = troubaire del v. 38: "Revenès, $o$ bèu cor, à la voues d'un troubaire», e, in prospettiva allargata, l'equazione felibri - trovatori, si attua all'interno di uno spazio metafisico digressivo, che confonde e coniuga le caratteristiche della visione con quelle del sogno:

«Pantaies? me veniéu à iéu-meme, o li masc t'an enclaus?» E lóugiero, à travès de l'ermas courrien, à pèd cauquet, de flamo de sant Ėume.

«Bèllis amo, disié Faneto de Gantèume, courtejaire valènt, troubadour chivalié, qu'avès mes en cansoun la divino foulié...»

[vv. 87-92]

18. Romanin, composta da 12 nobili donne, è - tra le Corti d'Amore inventate da Jean de Nostredame: Pierrefeu, Signe e Avignon— la Corte sovrana, quella più importante, alla quale finivano tutte le dispute irrisolte degli altri tribunali di dame.

19. L'edizione di riferimento è quella contenuta in Oeuvres complètes de Frédéric Mistral - Les Iles d'Or, texte et traduction, Marcel Petit, Raphèle-lès-Arles, 1980. 
Nell'enumerazione esemplare di trovatori e dame illustri medievali della strofa IV (vv. 121-122: "D'amour, diguè N'Alis la Coumtesso de Dìo, / enjusquo dins la mort lou pantai m'escandiho» ecc.), il sogno d'amore di matrice trobadorica (cioè, la fin'amors tradizionale) e il sogno mistraliano di una Provenza di nuovo felice e fiorente, sfociano in un lirismo bifronte - quello interiorizzante e quello archetipale, quello della poesia e quello della storiaprovocando quell' «insatisfaction» malinconica ${ }^{20}$ derivante dall'impossibile sutura dello spacco tra progettualità dell'io e applicabilità collettiva, con conseguente acquisizione dell' autocoscienza autoriale dell'illusorietà dell'immaginazione — nel monito «Remembras-vous...» del v. 126 — che disvela la propria consistenza di paradigma artificiale auto-prodotto; per cui il passo-chiave: «la vido èro un sounge» assume spessore tutto particolare per il fatto di essere pronunciato dal Monaco delle Isole d'Oro, fantomatico personaggio nostradamico: ${ }^{21}$

\section{[...] Dis Isclo d'Or lou Mounge}

diguè: «Remembras-vous que la vido èro un sounge!»

Pèire Vidau diguè: «Que i'ague quaucarèn

de plus dous que Prouvènço e qu'amour fugue rèn...

[vv. 125-28]

Le ambiguità irrisolte e irrisolvibili dell'universo lirico di Mistral si epitomizzano nel trittico tardo della raccolta Lis Oulivado (1912), ${ }^{22}$ dove l'ermeneutica del fantastico delinea tre assiomi monolitici della rappresentazione simbolica: l'archetipo (Lou Parangoun), il mito (il «fablèu» [v. 71] della Trevanço) e l'illusione (Lou Mirage). Il tutto applicato alla Provenza (Lou Parangoun, v. 23: «lou parangoun de ma Prouvènço bello»), come metafora socio-letteraria del sogno di rinascenza d'oc (Lou Parangoun, v. 7: «...lou pantai me sèmblo embruma d'or»), un'utopia a cui consacrare tutta la vita, con una non celata consapevolezza dei propri limiti (v. 6: «ma fe n'es qu'un pantai»), strutturalmente condensata — con inevitabile mise en relief sentenziosa - nella strofa conclusiva:

st. XII Basto: pèr iéu, sus la mar de l'istòri,

fuguères tu, Prouvènço, un pur simbèu,

20. Philippe Gardy, Frédéric Mistral et "Lou Pouèmo dóu Rose», "Annales de Littérature Occitane», 5 (1997), p. 42.

21. Il Moine des Isles d'Or è una delle fonti — dichiarata ma non svelata! — a cui Jean de Nostredame dice di rifarsi per i materiali e le informazioni delle sue Vies, p. 7: «Les vies de nos poëtes provençaux ont esté recueillies de plusieurs anciens autheurs, grands et excellens personnage, qui ont escript en nostre langue provençalle, et mesmes du Monge des Isles d'Or...». Sull'identificazione, a tutt'oggi non chiarita, di questo personaggio, cfr. R. CAPELLI, Anas vous..., art. cit., p. 448 , nota 22 .

22. L'edizione di riferimento è quella contenuta in Oeuvres complètes de Frédéric Mistral - Les Olivades, texte et traduction, Marcel Petit, Raphèle-lès-Arles, 1979. 
un miramen de glòri e de vitòri que, dins l'oumbrun di siècle transitòri, nous laisso vèire un eslùci dóu Bèu.

Non stupisce quindi che - con maggior approfondimento intimistico rispetto a Roumanin (1860) - un componimento come La Trevanço presenti un lessico del soprannaturale molto più denso, nel quale domina un' attenzione quasi ossessiva per la descrizione di epifanie dell'effimero, intangibili e spettrali, uno sciame di ombrucole e spiritelli (v. 1: "fantaumeto", v. 9: «oumbrinello», v. 13: «fatorgo antico», v. 14: «amo fantastico», v. 57: «la farfantello», v. 65: «oumbro») privi di qualunque consistenza, anche onirica, ridotti al rango di trasparenze sfocate del cuore (v. 45: "uno aparènço») o della memoria (v. 33: «uno souvènenço»), come è il riflesso della Provenza attuale rispetto al suo radioso passato:

\section{E la vido vidanto de-bado es abradanto: a respèt dóu fablèu, n'es qu'un rèire-soulèu.}

[vv. 69-72]

Se il sogno di Mistral tende, col tempo, ad identificarsi negativamente con il miraggio di un disegno di grandezza inattuabile, frustrante e frustrato:

Fanfarello d'amour, moun cor, que trop souvènt, li caresso despièi — e d'aqui me revèn tóuti li tentacioun de moun foulas jouvènt!...

\section{[Lou mirage, st. X]}

questo è perché la mise en scène del Medioevo è ancora troppo ingenua e la sublimazione retorica dei contenuti simbolici ancora troppo manifesta nei suoi procedimenti tecnico-formali: il trovatore Cercamon di Lou mirage è una persona (nel senso tecnico di "maschera» del teatro greco) alla maniera dei trovatori del giovane Pound o del Sordello di Browning, cioè una sorta di personificazione della voce fuori-campo dell'autore in un doppio attòrio, funzionale all'azione drammatica del testo più che alla definizione di un sentimento universale, che è poi l'essenza della "persona collettiva» connaturata all'esperienza onirica.

Questa ricerca di universalità si realizza nelle poesie di Max Rouquette, con l'annullamento dei ponti logici espliciti, abbandonando ogni similitudine a favore della metafora, giocando con le "paraulas coma paumas» [Castel d'Aumelas], capaci di accendere «la nuòch de las causas» [Las paraulas] e abbattere «la pòrta das sòmis» [Jol sause], per recuperare la «lenga perduda» [La lenga s'es perduda] ad una modernità iperstilizzata, che paradossalmente neutralizzi il 
peso del passato e della tradizione proprio attraverso la sua ipersemantizzazione. ${ }^{23}$ Così, la sestina L'Ataut d'Arnautz Daniel, cristallizza con l'ironia del pastiche arnaldiano ${ }^{24}$ il lascito metrico-numerologico del trovatore perigordino in un omaggio poetico alla sua celeberrima composizione, Lo ferm voler qu'el cor mintra, che relega alla dimensione del sogno le propaggini ormai svuotate di senso di qualunque revival trobadorico: ${ }^{25}$

Dieu garde oblit que jamai non t'oblida ni que son còr s'afana de l'orguòlh mon bel esper, ma lutz de plor de giure luna a solelh de l'eterne desir s'en gel e frech, los cavalhièrs de l'aura lo fuòc d'amor serva lo ram astruc.

D'ensenhas e de nuòch lo cap astruc e despulhat del còrs que tot oblida laura d'aram lo qu'amassava l'aura lo cant de flamba onte crema l'orguòlh onte se mòu d'estèla un blos desir en fosc camin de tenèbra e de giure.

De longs sospirs e de ròsas de giure floris l'ivern son sòmni benastruc velha la lebre e velha lo desir quand amargança ambe dolor s'oblida e que, rebat emporprat de l'orguòlh, als rams brancuts se derevèlha l'aura.

Au cant estrange onte mesclava a l'aura ranas de riu e bòsc nevat de giure rama de prima e sirèna d'orguòlh lo fol asard trevant lo cel astruc veniá, sutil aucèl qu'un vespre oblida pausar sa ròsa a tas bocas, desir.

23. Tutti i componimenti citati sono raccolti in Max ROUQUETTE, La maucòr de l'unicòrn / Le tourment de la licorne, (version française de l'auteur), Marseille: Sud Poésie, 1988.

24. Offro qui di sèguito un esempio, limitato alla seconda stanza, della tecnica di recupero e reimpasto creativo della matrice arnaldiana da parte di Rouquette:

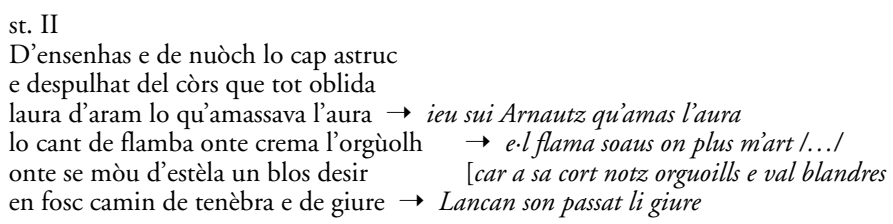

25. Sulla fortuna della sestina, cfr. Gabriele FrASCA, La furia della sintassi. La sestina in Italia, Napoli: Bibliopolis, 1992; e Pierre LARTIGUE, L'hélice d'écrire: la sextine, Paris: Les Belles Lettres, 1994. 
Sol que sàvia, coma l'aucèl desir

sul fuòc d'amor mescla sa cendra a l'aura

e puòi renais, lutz sus un temps qu'oblida

beu de la nuoch las lagremas de giure

e, cap levat dins lo matin astruc

trai dins lo cel las flambas de l'orguòlh.

Sorne solelh, astre roge, l'orguòlh, lavat tot lis a l'aiga del desir miralh de vida esclairat de l'astruc ombrat de ram escur, trevat de l'aura qu'esbeu lo temps coma beuriá de giure, l'orguòlh esbeu tot oblit e l'oblida.

Mon sòmi baile obrat d'orguòlh e d'aura au recòrd viu de desir e de giure d'Arnautz trobaire astruc que non s'oblida. ${ }^{26}$

La concatenazione morfo-sintattica assicurata dalla permutazione delle parole-rima, accentuata dal largo impiego di figure di parola e di pensiero, che producono l'effetto opposto e simultaneo di compressione eufonica del dettato poetico e dilatazione delle possibilità esegetiche del suo messaggio, gioca sui contrasti archetipici di giorno/notte (v. 29, lo matin astruc / v. 7, d'ensenhas e de nuòch; v. 11, d'estèla; v. 12, fosc camin de tenèbra; v. 23, vespre), e luce/ombra (vv. 3 e 27, lutz; v. 31, astre roge [ma riferito all'ossimorico sorne solelh]; v. 33, esclairat / v. 34, ombrat de ram escur), per confluire — come s'è visto- nel sogno del poeta; un sogno, per così dire, «ad occhi aperti», nel senso che quest'operare poetico è fortemente originale, è capace di affrancarsi dai modelli ingombranti di un passato vivo solo nel ricordo (v. 38: «recòrd viu»), che Rouquette riesce a seppellire (come annunciato dal titolo, dalla «tomba» di Arnaut Daniel) con gli stessi calembours e tours de force metrico-stilistici ${ }^{27}$ di un linguaggio tornato infine ai vertici della propria espressività.

26. Prima di essere inserito nella raccolta Lo maucòr de l'unicòrn..., cit. alla nota 23, p. 8-11, questo componimento era già stato pubblicato nella rivista $O c, 171$ (1949), p. 10-11.

27. Si noti, a titolo esemplificativo, l'associazione chiastica iper-significante nella tornada, che risulta dalle coppie incrociate e idealmente collegate tra loro di (v. 37) «mon sòmi» - «que non s'oblida» (v. 39) e (v. 39) «Arnautz» - «aura» (v. 37). 